\title{
Fixed-combination of amlodipine and diuretic chronotherapy in the treatment of essential hypertension: improved blood pressure control with bedtime dosing - a multicenter, open-label randomized study
}

\author{
Jing Zeng ${ }^{1,2}$, Min Jia ${ }^{1,2,5}$, Hua Ran ${ }^{3,5}$, Hui Tang ${ }^{4,5}$, Ye Zhang ${ }^{1,2}$, Jun Zhang ${ }^{1,2}$, Xukai Wang ${ }^{1,2}$, \\ Hongyong Wang ${ }^{1,2}$, Chengming Yang ${ }^{1,2}$ and Chunyu Zeng ${ }^{1,2}$
}

Previous studies have demonstrated that individual anti-hypertension medications have different effects when administered in the morning vs. the evening. However, the impact of administration timing on fixed combinations of anti-hypertensive medications on blood pressure control is still unknown. In the present study, we examined the administration time-dependent effects of a fixed combination of amlodipine and diuretics (amlodipine complex) on blood pressure in hypertensive subjects. Eighty patients from Chongqing City were enrolled in this study. Subjects were randomly assigned to receive a single pill (amlodipine complex, each tablet containing amlodipine $5 \mathrm{mg}$ and hydrochlorothiazide $25 \mathrm{mg}$ ), either in the morning (0800 hours, $n=40$ ) or at bedtime ( 2200 hours, $n=40$ ). Blood pressure was measured by ambulatory monitoring every 20 min during the day and every $30 \mathrm{~min}$ at night for 24 consecutive hours before and after the 12 weeks of treatment. Following treatment, the 24-h mean systolic and diastolic blood pressures were reduced significantly in both the morning and bedtime groups. However, the morning blood pressure surge was reduced to a greater degree in the bedtime group. In addition, the nocturnal blood pressure and the $24 \mathrm{~h}$ mean blood pressure were lower in the bedtime group. More patients converted from having a non-dipper to dipper blood pressure in the bedtime group. These findings confirm that amlodipine complexes have different efficiencies depending on treatment time. Administration of amlodipine complexes at bedtime could optimize the anti-hypertensive effect by augmenting blood pressure-lowering effects, increasing the diurnal/nocturnal ratio of blood pressure, normalizing the blood pressure pattern and minimizing the morning blood pressure surge.

Hypertension Research (2011) 34, 767-772; doi:10.1038/hr.2011.36; published online 7 April 2011

Keywords: amlodipine; chronotherapy; hydrochlorothiazide

\section{INTRODUCTION}

Hypertension, a common risk factor for cardiovascular morbidity and mortality, is highly prevalent, affecting $\sim$ one billion individuals worldwide. ${ }^{1,2}$ Over the years, concomitant with an increased understanding of the epidemiology of hypertension and the beneficial effects of treatment, there has been a progressive lowering of blood pressure values in treated hypertensives towards optimal targets. Even though awareness and treatment of hypertension have increased, substantial improvements in blood pressure control rates are still needed. ${ }^{3,4}$ Currently, it is postulated that hypertension should be considered a multifactorial disease, often requiring multiple drugs for its manage- ment, with the majority of hypertensive patients requiring two or more agents to reach a specific blood pressure goal, particularly for those patients with stage 2 or stage 3 hypertension. ${ }^{2-4}$ The guidelines in the Seventh Report of the Joint National Committee on Prevention, Detection, Evaluation, and Treatment of High Blood Pressure (JNC VII $)^{5}$ recommend the use of fixed combinations of antihypertensive medications, including calcium channel blockers (CCBs) and diuretics. ${ }^{6}$

Besides blood pressure level, blood pressure characteristics, including blood pressure pattern, morning surge and the diurnal/nocturnal blood pressure ratio are all independent risk factors for cardiovascular

${ }^{1}$ Department of Cardiology, Cardiovascular Hospital in Daping Hospital, The Third Military Medical University, Chongqing, PR China; ${ }^{2}$ Chongqing Institute of Cardiology, Chongqing, PR China; ${ }^{3}$ Department of Cardiology, Central Hospital of Qianjiang City, Chongqing City, PR China and ${ }^{4}$ Department of Cardiology, Shuangqiao District Hospital, Chongqing, PR China

${ }^{5}$ Co-first authors.

Correspondence: Dr C Zeng, Department of Cardiology, Daping Hospital, The Third Military Medical University, Chongqing City, PR China.

E-mail: cyzeng1@hotmail.com

Received 28 October 2010; revised 22 December 2010; accepted 26 January 2011; published online 7 April 2011 
disease (CVD).$^{7-9}$ Normalization of the circadian rhythm of blood pressure is considered to be an important clinical goal of pharmacotherapy. Due to the time-dependent kinetics of medications, as well as normal circadian rhythms during drug-free times, and the rate-limiting steps of key metabolic pathways, it is not surprising that anti-hypertensive medications would display circadian-dependent effects. Previous studies have demonstrated differing effects of angiotensin-converting enzyme inhibitors, CCBs and diuretics when administered in the morning vs. the evening. ${ }^{10-13}$ However, rarely has the time of drug administration been a specific investigative focus for fixed combinations of anti-hypertensive medications. This study compared, by $24-\mathrm{h}$ ambulatory blood pressure monitoring (ABPM), the anti-hypertensive efficacy of the administration of a fixed combination of amlodipine (a CCB) and a diuretic (hydrochlorothiazide) in a single pill (amlodipine complex) in the morning $v$ s. the evening over a 12 -week period.

\section{METHODS}

\section{Patient selection}

The present study was a multicenter open-label randomized trial on the effects of morning vs. evening administration of an amlodipine complex on ambulatory blood pressure. The subjects included Chinese hypertensive patients recruited from three Chinese hospital clinics. The entry period was from March 2008 to June 2009 . We studied 80 subjects ( 43 men and 37 women), $67.8 \pm 9.8$ years of age, with essential hypertension (stages 1-2), as defined by the JNC $\mathrm{VII}^{5}$ guidelines. The determination of hypertension stage was based on conventional blood pressure measurements and corroboration by ABPM at the time of recruitment. The prerequisite for inclusion was documented hypertension on three separate visits before enrollment. A patient was diagnosed with hypertension if the mean of three sphygmomanometric blood pressure readings exceeded $140 / 90 \mathrm{~mm} \mathrm{Hg}$ and the patient was not taking any anti-hypertensive medication. A positive diagnosis of hypertension based on ABPM required that the 24-h mean patient systolic blood pressure (SBP)/ diastolic blood pressure (DBP) was greater than $130 / 80 \mathrm{~mm} \mathrm{Hg}$, the diurnal mean was greater than $135 / 85 \mathrm{~mm} \mathrm{Hg}$, or the nocturnal mean was greater than $125 / 75 \mathrm{~mm} \mathrm{Hg} .^{5}$ All subjects received their routine medical care from the Department of Cardiology at three hospitals in Chongqing City. Key exclusion criteria were any known form of secondary hypertension, bradycardia (heart rate (HR) $<45$ beats per min), tachycardia (HR $>100$ beats per min), stroke or myocardial infarction in the preceding 6 months, congestive heart failure (left ventricular ejection fraction $<40 \%$ ), clinically significant hepatic or renal disease, uncontrolled diabetes mellitus, chronic obstructive pulmonary disease, life-style factors such as night-shift work, history of drug and alcohol abuse, neurological and psychiatric illnesses, and pregnancy or breast-feeding. The study protocol was approved by the ethics committees of the three recruitment hospitals, and all subjects provided informed consent.

After providing informed consent to participate in this open-label, randomized, chronotherapy trial, there was a 2-week washout period when required (17.5\% of the participants had not been treated previously for hypertension and an additional $37.5 \%$ were untreated for at least 6 months). Following the washout period, all remaining subjects were randomly assigned to receive a single amlodipine complex pill, which contained $5 \mathrm{mg}$ of amlodipine and $25 \mathrm{mg}$ of hydrochlorothiazide (Dongrui Pharmaceuticals Company, Jiangsu Province, China), either in the morning (0800 hours) or at bedtime (2200 hours). If the blood pressure goal (office blood pressure $<140 / 90$ ) was not achieved, the dosage of medicine was titrated to 1.5 or 2 pills. The demographic characteristics of the participants, including any previous use of anti-hypertensive medications, are described in Table 1.

Blood samples were obtained in the clinic from an antecubital vein after nocturnal fasting. All samples were taken between 0800 and 0900 hours immediately before treatment on the same day the 24-h ABPM was initiated. Conventional office blood pressure measurements were always obtained by the same investigator to avoid potential observer bias effects. The assignment of subjects to treatment groups was done by one member of the research team, according to the order of recruitment, following an allocation table constructed by a computerized random-number generator. The assignment of subjects to
Table 1 Basal characteristics of hypertensive patients

\begin{tabular}{lccc}
\hline & $\begin{array}{c}\text { Dosing in } \\
\text { the morning }\end{array}$ & $\begin{array}{c}\text { Dosing at } \\
\text { bedtime }\end{array}$ & P-value \\
\hline Patient number & 40 & 40 & NS \\
Never treated $(n)$ & 7 & 8 & NS \\
Male (\%) & $43.2 \%$ & $40.7 \%$ & NS \\
Age (years) & $66.9 \pm 9.3$ & $68.5 \pm 10.0$ & NS
\end{tabular}

Complication ( $\mathrm{n}$ )

CAD

Diabetes

Hyperlipidemia

9

4

11

7

6

9

NS

NS

NS

Previously used medicine (n)

$\mathrm{CCB}$

Diuretic

ACEI

ARB

$\beta$-receptor blocker

BMI $\left(\mathrm{kg} \mathrm{m}^{-2}\right)$

$\mathrm{FG}\left(\mathrm{mmoll}^{-1}\right)$

Triglycerides $\left(\mathrm{mmoll}^{-1}\right)$

Total cholesterol ( $\left.\mathrm{mmol} \mathrm{I}^{-1}\right)$

Urine protein positive rate (\%)

Creatinine $\left(\mu \mathrm{mol} \mathrm{I}^{-1}\right)$

GOT $\left(\mathrm{UI}^{-1}\right)$

GPT $\left(U I^{-1}\right)$

Uric acid $\left(\mu \mathrm{mol} \mathrm{I}^{-1}\right)$

Office BP (SBP/DBP, $\mathrm{mm} \mathrm{Hg}$ )

$\begin{array}{ccc}23 & 25 & \text { NS } \\ 3 & 2 & \text { NS } \\ 7 & 5 & \text { NS } \\ 5 & 4 & \text { NS } \\ 6 & 7 & \text { NS } \\ 25.5 \pm 3.8 & 24.6 \pm 4.1 & \text { NS } \\ 5.3 \pm 0.8 & 5.4 \pm 0.9 & \text { NS } \\ 1.95 \pm 0.73 & 1.98 \pm 1.59 & \text { NS } \\ 4.92 \pm 0.85 & 4.63 \pm 0.97 & \text { NS } \\ 10 \% & 15 \% & \text { NS } \\ 80.1 \pm 16.6 & 74.5 \pm 18.3 & \text { NS } \\ 31.5 \pm 15.1 & 30.2 \pm 21.6 & \text { NS } \\ 28.3 \pm 8.0 & 28.8 \pm 12.3 & \text { NS } \\ 378.1 \pm 88.2 & 327.9 \pm 78.6 & \text { NS } \\ 158.5 \pm 9.2 / & 155.6 \pm 9.7 / & \text { NS } \\ 92.4 \pm 7.8 & 92.2 \pm 6.5 & \end{array}$

Abbreviations: $\mathrm{ACEI}$, angiotensin converting enzyme inhibitor; $\mathrm{ARB}, \mathrm{AT}_{1}$ receptor blocker; $\mathrm{BMI}$ body mass index; BP, blood pressure; $C A D$, coronary artery disease;

$\mathrm{CCB}$, calcium channel blocker; DBP, diastolic blood pressure; FG, fasting serum glucose; GOT, glutamic oxalacetic transaminase; GPT, glutamate-pyruvate transaminase; $n$, number of patients; NS, no significant difference; SBP, systolic blood pressure.

their respective treatment-time groups was blinded from both the research team member conducting the conventional blood pressure measurements and from the team members performing the statistical analysis of the data.

\section{Blood pressure assessment}

The SBP, DBP and HR of each participant were automatically measured every 20 min during the day (0600-2200 hours) and every 30 min during the night for 24 consecutive hours ${ }^{14,15}$ with an ABPM device (Wuxi Zhongjian, Wuxi City, China). All subjects were examined by ABPM under baseline conditions when they were free of medication (after the 2-week washout for previously treated subjects) both before and after the 12 weeks of timed therapy. The blood pressure of the subjects was assessed while they adhered to their usual diurnal activity schedule (0600-2200 hours for most) and nocturnal sleep routines. Participants were instructed to go about their usual activities with minimal restrictions but were instructed to follow a similar schedule during the day of ABPM. No one was hospitalized during the study period. ABPM was always initiated between 1000-1200 hours. Blood pressures or subjects were eliminated from the analysis when $>30 \%$ of the measurements were missing, there were missing data for a $>3 \mathrm{~h}$ span, or the subject had a night time sleep span $<6 \mathrm{~h}$ or $>12 \mathrm{~h}$ during the monitoring period.

Non-dippers were defined as those who did not exhibit a reduction in mean SBP of $\geqslant 10 \mathrm{~mm} \mathrm{Hg}$ from daytime to night time. All remaining subjects were classified as dippers. The overall smoothness index was defined as the average of hourly mean blood pressure reductions divided by the s.d. of the average. The trough-to-peak ratio was defined as the average blood pressure reduction during the last $2 \mathrm{~h}$ of the dosing interval compared with the average of the maximal reduction in blood pressure over 2 consecutive hours. 
Statistical analysis

Data are expressed as mean \pm s.d. Comparison within groups was made by repeated measures analysis of variance, and comparison among groups was performed by factorial analysis of variance and Duncan's test ( $t$-test when only two groups were compared). A value of $P<0.05$ was considered significant.

\section{RESULTS}

\section{Subject characteristics}

The baseline characteristics of the two subject groups (Table 1) were comparable in age, body mass index, SBP and DBP (average of the conventional morning measurements obtained just before ABPM). Moreover, there were no statistically significant changes in weight, body mass index, and waist and hip circumferences in any patient after 12 weeks of timed treatment (data not shown). The serum values of fasting glucose, creatinine, cholesterol, triglycerides, uric acid (Table 1) and other laboratory-measured metabolites in the two treatment groups were comparable at baseline and were unchanged after 12 weeks of treatment (data not shown).

\section{Amlodipine complex on awakening}

The circadian rhythm of SBP and DBP, as measured by 24-h ABPM before and after 12 weeks of treatment with amlodipine complex upon awakening, is depicted in Figures $1 \mathrm{a}$ and $\mathrm{b}$. Dosing in the morning resulted in a statistically significant reduction in blood pressure from baseline after 12 weeks of treatment $(P<0.001)$. After 12 weeks of treatment, ABPM showed that $75 \%$ of the subjects had diurnal, nocturnal, and 24-h SDP and DBP means below the respective thresholds for a diagnosis of hypertension. Moreover, all patients had a $>10 \%$ reduction in their baseline 24 -h mean blood pressure.

The trough-to-peak ratio was 55.2 and $57.3 \%$ for SBP and DBP, respectively, following dosing of amlodipine complex upon awakening. The overall smoothness index was also very high $(0.98 \pm 0.23$ and $1.27 \pm 0.35$ for SBP and DBP, respectively), indicating a similar blood pressure reduction during the entire $24 \mathrm{~h}$ period. Figure 1 further indicates that the mean reduction in blood pressure at each measured time point during the 24-h dosing interval was statistically significant ( $P$ always $<0.005)$. Despite the highly significant reduction in blood pressure, no effect of amlodipine complex on HR was observed (Table 2).

\section{Amlodipine complex at bedtime}

Table 2, Figures $1 \mathrm{a}$ and $\mathrm{b}$ show a significant reduction in the 24-h mean of SBP and DBP, respectively $(P<0.001)$ after 12 weeks of amlodipine complex being taken at bedtime. The percentage of subjects with controlled blood pressure according to ABPM criteria was $80 \%$ in the bedtime group. All patients showed a $>10 \%$ reduction in the 24-h mean blood pressure. Despite the significant effects of treatment observed on blood pressure, HR remained unchanged after 12 weeks of treatment (increase in the 24-h mean was 0.8 beat per minute; $P>0.05$ ) (Table 2).

The blood pressure reduction was statistically significant $(P$ always $<0.005$ ) at each of the measured times during the 24 -h period, as shown in Figures $1 \mathrm{a}$ and $\mathrm{b}$. These results indicate that administration of the amlodipine complex at bedtime had a greater blood pressurelowering effect throughout the entire 24-h dosing interval than when administered in the morning upon awakening. The bedtime trough-topeak ratio was also higher than the morning observed ratio following administration of amlodipine complex upon awakening (63.7 and $66.9 \%$ for SBP and DBP, respectively). The observed smoothness index was also higher when amlodipine complex was administered at bedtime (1.53 \pm 0.62 and $1.71 \pm 0.68$ for SBP and DBP, respectively).
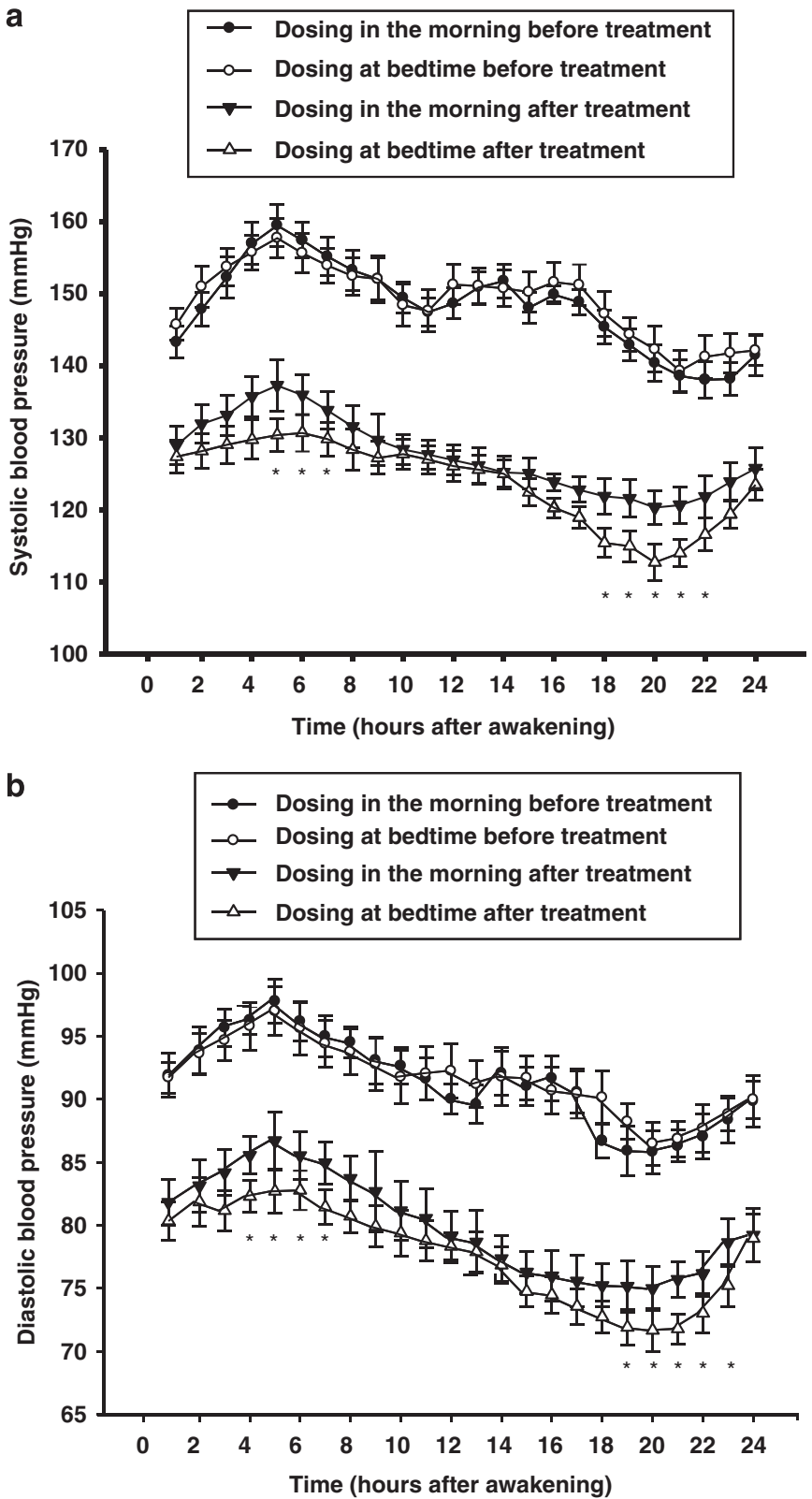

Figure 1 Effects on systolic blood pressure (SBP) (a) and diastolic blood pressure (DBP) (b) measured by 24-h ambulatory blood pressure monitoring (ABPM) in subjects with stage 1 or 2 essential hypertension before and after 12 weeks of treatment with an amlodipine complex administered either in the morning or at bedtime. All results are expressed as $\mathrm{mm} \mathrm{Hg}(n=40$, ${ }^{*} P<0.01$ vs. dosing in the morning, analysis of variance, Duncan's test).

\section{Comparison between groups}

The comparison of results, as shown in Table 1, reveals lack of statistically significant differences in ambulatory blood pressure at baseline between the two treatment groups. After treatment for 12 weeks, the diurnal mean blood pressure tended to be lower in the group taking the amlodipine complex at bedtime than in the group taking the amlodipine complex in the morning, although statistical significance was not achieved (Figures 2a and b). However, the 24-h mean blood pressure and nocturnal mean blood pressure (Table 2, Figures $2 \mathrm{a}$ and $\mathrm{b}$ ) were significantly lower in the group taking the amlodipine complex at bedtime than in the group taking the amlo- 
Table 2 Blood pressure and heart rate in both groups before and after treatment

\begin{tabular}{lcc}
\hline & $\begin{array}{c}\text { Dosing in the morning } \\
(\mathrm{n}=40)\end{array}$ & $\begin{array}{c}\text { Dosing at bedtime } \\
(\mathrm{n}=40)\end{array}$ \\
\hline Before treatment & & \\
$24 \mathrm{~h}$ mean BP & & \\
SBP & $149.7 \pm 17.7$ & $147.8 \pm 18.5$ \\
DBP & $92.7 \pm 15.22$ & $92.6 \pm 11.2$ \\
Non-dipper $(\%, n)$ & $53 \%(21)$ & $55 \%(22)$ \\
Mean HR (beats per min) & $75.0 \pm 6.6$ & $73.6 \pm 7.7$ \\
& & \\
After treatment & & $121.6 \pm 13.7^{*, * *}$ \\
24h mean BP & & $75.5 \pm 9.12^{*, * *}$ \\
SBP & $127.5 \pm 15.22^{*}$ & $30 \%(12)^{*, * *}$ \\
DBP & $81.0 \pm 8.07^{*}$ & $72.8 \pm 6.2$ \\
Non-dipper (\%, $n$ ) & $45 \%(18)$ & \\
Mean HR (beats per min) & $75.8 \pm 6.5$ & \\
\hline
\end{tabular}

Abbreviations: BP, blood pressure; DBP, diastolic blood pressure; HR, heart rate; SBP, systolic blood pressure.

${ }^{*} P<0.05$ vs. before treatment; ${ }^{* *} P<0.05$ vs. dosing in the morning.

dipine complex in the morning. Accordingly, there was a highly significantly greater increase $(P<0.001)$ in the nocturnal decline in blood pressure relative to the diurnal mean (an index of blood pressure dipping) when amlodipine complex was taken at bedtime (Figures $2 \mathrm{a}$ and $\mathrm{b}$ ). The reduction in the frequency of non-dippers was only $8.0 \%$ in the morning group. However, the reduction $(25.0 \%)$ in the frequency of subjects who at baseline were non-dippers was highly significant $(P<0.001)$ when the same dose of amlodipine complex was taken at bedtime for 12 weeks (Table 2).

We also evaluated the effect of treatment time on the diurnal/ nocturnal ratio of blood pressure and the slope of morning rise in blood pressure. We found no significant changes in the slope of the morning rise or nocturnal decline for either SBP or DBP when amlodipine complex was taken upon awakening. However, when the amlodipine complex was taken at bedtime, there was a lesser increase in the slope of the morning rise and a greater increase in the slope of the evening decline in SBP and DBP (Figures 1a and b).

\section{DISCUSSION}

To the best of our knowledge, the present study is the first to examine how a fixed combination of an anti-hypertensive medicine, amlodipine complex, had a difference in efficacy depending on the time of drug administration. Administration in the evening at bedtime, relative to administration in the morning, minimized the morning blood pressure surge and enhanced both the evening decline and the reduction in the nocturnal blood pressure. More non-dipper patients were converted into dipper status in the bedtime group.

The clinically relevant dosing-time differences in the beneficial and adverse effects of blood pressure-lowering medications are known. ${ }^{9,16}$ Previous studies have investigated the chronotherapeutics of several medicines, including diuretics, angiotensin converting enzyme inhibitors and $\mathrm{AT}_{1}$ receptor blockers and CCBs. ${ }^{10-13}$ Studied CCBs include amlodipine, ${ }^{10,17}$ diltiazem, ${ }^{11}$ and cilnidipine. ${ }^{18}$ Portaluppi et al. ${ }^{19}$ explored the relative advantage of evening vs. morning once-a-day treatment with a conventional sustained-release isradipine formulation in non-dipper hypertensive patients with chronic renal failure and found that an evening dosing schedule best reduces and normalizes nocturnal SBP and DBP. However, not all calcium-channel
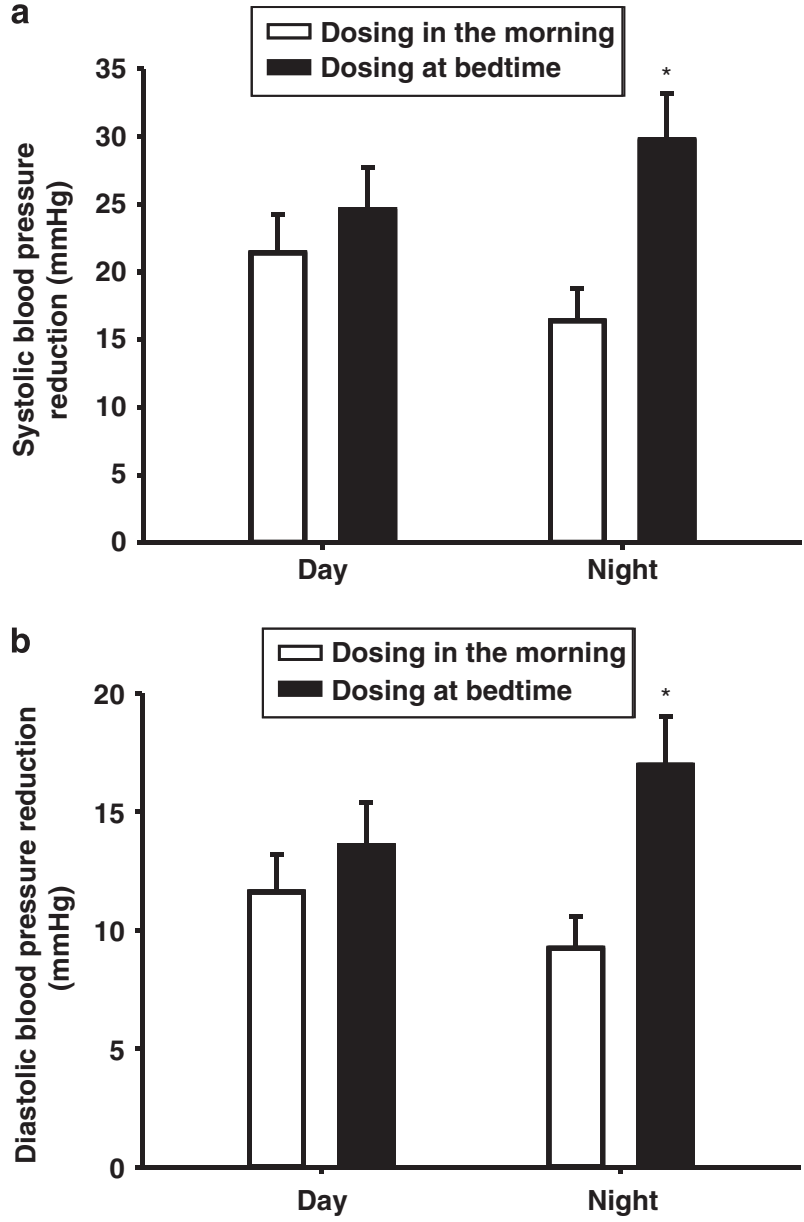

Figure 2 Absolute decrease in systolic blood pressure (a) and diastolic blood pressure (b) measured by 24-h ambulatory monitoring in subjects with stage 1 or 2 essential hypertension after 12 weeks of treatment with amlodipine complex administered upon awakening or at bedtime. All results are expressed as $\mathrm{mm} \mathrm{Hg}\left(n=40,{ }^{*} P<0.01\right.$ vs. dosing in the morning, paired $t$ test).

blockers exhibit different efficacies with different dosing-times. Kitahara et al. ${ }^{18}$ did not find a differential dosing-time effect on cilnidipine during an open, randomized cross-over study. ${ }^{18}$ A loop diuretic (torasemide) given in the morning or at bedtime was found to be more efficacious when administered at bedtime. The time-response curves also indicated complete 24 -h therapeutic coverage only when torasemide was administered at bedtime. The percentage of patients with controlled ambulatory blood pressure was also significantly greater with the bedtime dosing schedule compared with morning dosing. $^{20}$

Meticulous control of blood pressure is required in hypertensive patients to produce the maximum reduction toward clinical cardiovascular end points. Recent clinical trials suggest that a monotherapy approach for the control of hypertension is not likely to be successful in most patients. Combination therapy may be theoretically favored due to the fact that multiple factors contribute to hypertension, and achieving control of blood pressure with a single agent acting through one particular mechanism may not be possible. ${ }^{21}$ Regimens can either be fixed-dose combinations or drugs taken sequentially. Diuretics and CCBs are effective in reducing blood pressure. ${ }^{22}$ As mentioned before, previous studies have demonstrated different effects of CCBs or 
diuretics when dosed in the morning vs. the evening. However, whether fixed combinations of anti-hypertensive medications have circadian effect variations is not known. Our present study found, consistent with the results observed in monotherapy studies, administration of an amlodipine complex at bedtime reduced the $24 \mathrm{~h}$ mean SBP/DBP and nocturnal blood pressure to a greater degree than administration in the morning. There was an observed tendency toward lower mean daytime blood pressure in the bedtime administration group compared with the morning administration group. However, this difference was not statistically significant. The lack of statistical significance may be related to the small sample size of the study. Therefore, the results of this study need to be confirmed in a clinical trial with a larger sample size.

The circadian pattern of blood pressure is typically characterized by an increase in the early morning and a decrease during sleep. Nondipper hypertension is associated with an elevated risk of end-organ injury, particularly to the heart, brain and kidney. ${ }^{23-25}$ Ohkubo et al., who followed patients in Japan for more than 9 years, ${ }^{24}$ found a linear and inverse relationship between cardiovascular mortality and nocturnal blood pressure decline. Verdecchia et al..$^{23}$ also demonstrated that non-dipper hypertensive patients experience nearly three times as many adverse cardiovascular events as dippers. Analysis of a subgroup of 808 subjects in the Syst-Eur trial found that non-dippers experience a greater incidence of stroke and myocardial infarction than dippers. ${ }^{24}$ After an average follow-up of 9.2 years, a 5\% decrease in the decline of nocturnal SBP in hypertensive patients was associated with a $31 \%$ increased risk of cardiovascular mortality. ${ }^{24}$ The above-mentioned results indicate that cardiovascular risk could be influenced not only by blood pressure elevation but also by circadian variability in blood pressure. However, not all anti-hypertensive medicines have beneficial effects on blood pressure pattern. Hermida et al. reported that antihypertensive drugs given in the morning increased the prevalence of non-dipping blood pressure from 38 to $62 \%$ in resistant hypertensive patients. $^{26}$ Thus, the best method of tailoring the treatment of nondippers is a major concern of chronotherapy. Uzu and Kimura ${ }^{27}$ reported that hydrochlorothiazide restores the diminished nocturnal blood pressure decline, and shifts circadian rhythm of blood pressure from a non-dipper to a dipper profile. Hermida et al. ${ }^{28}$ reported that valsartan administration at bedtime, as opposed to administration upon awakening, resulted in a highly significant average increase in the diurnal/nocturnal ratio of blood pressure, corresponding to a $73 \%$ relative reduction in the number of non-dipper patients. In another study, the bedtime administration of trandolapril also significantly reduced morning blood pressure levels. ${ }^{29}$

Besides the circadian blood pressure pattern, increasing numbers of studies indicate that cardiovascular events occur more frequently in the morning, and that this phenomenon may be related to the morning blood pressure surge. ${ }^{30}$ An anti-hypertensive medication that reduces the morning blood pressure surge would be useful in the prevention of cardiovascular events in hypertensive patients. The present study, the first prospective randomized trial to focus on a fixed combination of two anti-hypertensive medicines, found that similar to the chronotherapy studies of single medications, administration of amlodipine complex at bedtime significantly minimizes the morning surge, lowers the nocturnal blood pressure, increases the diurnal/ nocturnal ratio of blood pressure and reduces the number of nondipper patients.

\section{Perspectives}

Chronotherapeutics aims to proportion the serum and tissue concentrations of medicines in synchrony with known circadian rhythms.
Previous studies have demonstrated the differing effects of single anti-hypertensive medication following morning $v s$. evening dosing. ${ }^{17-20,31,32}$ The retrospective Syst-Eur ${ }^{25}$ and HOPE $^{33}$ outcome trials show that evening administration of nitrendipine and ramipril impacts sleep-time blood pressure by converting the 24-h blood pressure pattern to a dipper pattern and decreasing CVD risk in the HOPE study. The CONVINCE trial ${ }^{34,35}$ did not show a chronotherapy benefit, but the MAPEC trial ${ }^{36}$ results suggest that night time dosing is beneficial. The CONVINCE trial intended to compare CVD protection afforded by conventional $\beta$-blocker and diuretic medications $v s$. a special drug-delivery verapamil formulation as a bedtime hypertension chronotherapy. However, the trial was terminated prematurely because of a corporate business decision, not based on inadequate performance of the chronotherapy. The MAPEC study was a prospective study to test the hypothesis that bedtime administration of more than one conventional medication exerts better blood pressure control and CVD risk reduction than the traditional approach of scheduling all medications in the morning. The results of this 5.6-year median follow-up study established that bedtime chronotherapy more effectively improves blood pressure control, better decreases prevalence of non-dipping patients, and, most importantly, reduces CVD morbidity and mortality. However, whether a fixed combination of anti-hypertensive medications, specifically a combination of amlodipine and hydrochlorothiazide, has any longterm protective effects on end-organ injury remains to be investigated.

\section{ACKNOWLEDGEMENTS}

These studies were supported, in part, by grants from the National Natural Science Foundation of China (30470728, 30672199), Natural Science Foundation Project of CQ CSTC (CSTC,2009BA5044), and grants for Distinguished Young Scholars of China from the National Natural Science Foundation of China (30925018).

1 Lee DE, Cooper RS. Recommendations for global hypertension monitoring and prevention. Curr Hypertens Rep 2009; 11: 444-449.

2 He FJ, MacGregor GA. Reducing population salt intake worldwide: from evidence to implementation. Prog Cardiovasc Dis 2010; 52: 363-382.

3 Egan BM, Zhao Y, Axon RN. US trends in prevalence, awareness, treatment, and control of hypertension, 1988-2008. JAMA 2010; 303: 2043-2050.

4 Oparil S, Weber M. Angiotensin receptor blocker and dihydropyridine calcium channel blocker combinations: an emerging strategy in hypertension therapy. Postgrad Med 2009; 121: 25-39.

5 Chobanian AV, Bakris GL, Black HR, Cushman WC, Green LA, Izzo Jr JL, Jones DW, Materson BJ, Oparil S, Wright Jr JT, Roccella EJ, Joint National Committee on Prevention, Detection, Evaluation, and Treatment of High Blood Pressure; National Heart, Lung, and Blood Institute; National High Blood Pressure Education Program Coordinating Committee. Seventh report of the Joint National Committee on Prevention, Detection, Evaluation, and Treatment of High Blood Pressure. Hypertension 2003; 42: 1206-12052.

6 The World Health Report, 2002. Reducing Risks, Promoting Healthy Life. World Health Organization: Geneva, Switzerland. 2002: 58.

7 Kario K, Pickering TG, Umeda Y, Hoshide S, Hoshide Y, Morinari M, Murata M, Kuroda T, Schwartz JE, Shimada K. Morning surge in blood pressure as a predictor of silent and clinical cerebrovascular disease in elderly hypertensives: a prospective study. Circulation 2003; 107: 1401-1406.

8 Kaya MG, Yarlioglues M, Gunebakmaz O, Gunturk E, Inanc T, Dogan A, Kalay N, Topsakal R. Platelet activation and inflammatory response in patients with non-dipper hypertension. Atherosclerosis 2010; 209: 278-282.

9 Hermida RC, Ayala DE, Calvo C, Portaluppi F, Smolensky MH. Chronotherapy of hypertension: Administration-time-dependent effects of treatment on the circadian pattern of blood pressure. Adv Drug Deliv Rev 2007; 59: 923-939.

10 Nold G, Strobel G, Lemmer B. Morning vs evening amlodipine treatment: effect on circadian blood pressure profile in essential hypertensive patients. Blood Press Monit 1998; 3: 17-25.

11 Kohno I, Iwasaki H, Okutani M, Mochizuki Y, Sano S, Satoh Y, Ishihara T, Ishii H, Mukaiyama S, Ijiri H, Komori S, Tamura K. Administration-time-dependent effects of diltiazem on the 24-h blood pressure profile of essential hypertension patients. Chronobiol Int 1997; 14: 71-84. 
12 Hermida RC, Ayala DE, Mojón A, Chayán L, Domínguez MJ, Fontao MJ, Soler R, Alonso I, Fernandez JR. Comparison of the effects on ambulatory blood pressure of awakening versus bedtime administration of torasemide in essential hypertension. Chronobiol Int 2008; 25: 950-970.

13 Hermida RC, Ayala DE, Fernández JR, Calvo C. Comparison of the efficacy of morning versus evening administration of telmisartan in essential hypertension. Hypertension 2007; 50: 715-722.

14 Stergiou GS, Nasothimiou EG, Roussias LG. Morning hypertension assessed by home or ambulatory monitoring: different aspects of the same phenomenon? J Hypertens 2010; 28: 1846-1853.

15 Hermida RC, Ayala DE, Fontao MJ, Mojón A, Alonso I, Fernández JR. Administrationtime-dependent effects of spirapril on ambulatory blood pressure in uncomplicated essential hypertension. Chronobiol Int 2010; 27: 560-574.

16 Smolensky MH, Hermida RC, Ayala DE, Tiseo R, Portaluppi F. Administration-timedependent effects of blood pressure-lowering medications: basis for the chronotherapy of hypertension. Blood Press Monit 2010; 15: 173-180.

17 Mengden T, Binswanger B, Gruene S. Dynamics of drug compliance and 24-h blood pressure control of once daily morning vs evening amlodipine. J Hypertens 1992; 10 (Suppl 4): S136.

18 Kitahara Y, Saito F, Akao M, Fujita H, Takahashi A, Taguchi H, Hino T, Otsuka Y, Kushiro $\mathrm{T}$, Kanmatsuse $\mathrm{K}$. Effect of morning and bedtime dosing with cilnidipine on blood pressure, heart rate, and sympathetic nervous activity in essential hypertensive patients. J Cardiovasc Pharmacol 2004; 43: 68-73.

19 Portaluppi F, Vergnani L, Manfredini R, degli Uberti EC, Fersini C. Time-dependent effect of isradipine on the nocturnal hypertension in chronic renal failure. Am J Hypertens 1995; 8: 719-726.

20 Calvo C, Hermida RC, Ayala DE, López JE, Rodríguez M, Chayán L, Mojón A, Soler R, Fontao MJ, Fernández JR. Chronotherapy with torasemide in hypertensive patients: increased efficacy and therapeutic coverage with bedtime administration. Med Clin (Barc) 2006; 127: 721-729.

21 Chobanian AV, Bakris GL, Black HR, Cushman WC, Green LA, Izzo Jr JL, Jones DW, Materson BJ, Oparil S, Wright Jr JT, Roccella EJ, National Heart, Lung, and Blood Institute; Joint National Committee on Prevention, Detection, Evaluation, and Treatment of High Blood Pressure; National High Blood Pressure Education Program Coordinating Committee. The Seventh Report of the Joint National Committee on Prevention, Detection, Evaluation, and Treatment of High Blood Pressure: the JNC 7 report. JAMA 2003; 289: 2560-2572.

22 Hassler C, Burnier M. Circadian variations in blood pressure: implications for chronotherapeutics. Am J Cardiovasc Drugs 2005; 5: 7-15.

23 Verdecchia P, Porcellati C, Schillaci G, Borgioni C, Ciucci A, Battistell M, Guerrieri M, Gatteschi C, Zampi I, Santucci A, Santucci C, Reboldi G. Ambulatory blood pressure: an independent predictor of prognosis in essential hypertension. Hypertension 1994; 24: 793-801.

24 Ohkubo T, Hozawa A, Yamaguchi J, Kikuya M, Ohmori K, Michimata M, Matsubara M, Hashimoto J, Hoshi H, Araki T, Tsuji I, Satoh H, Hisamichi S, Imai Y. Prognostic significance of the nocturnal decline in blood pressure in individuals with and without high 24-h blood pressure: the Ohasama study. J Hypertens 2002; 20: 2183-2189.

25 Staessen JA, Thijs L, Fagard R, O'Brien ET, Clement D, de Leeuw PW, Mancia G, Nachev C, Palatini P, Parati G, Tuomilehto J, Webster J, Systolic Hypertension in Europe (Syst-Eur) trial investigators. Predicting cardiovascular risk using conventional vs ambulatory blood pressure in older patients with systolic hypertension. JAMA 1999; 282: 539-546.

26 Hermida RC, Ayala DE, Calvo C, López JE, Mojón A, Fontao MJ, Soler R, Fernández JR. Effects of time of day of treatment on ambulatory blood pressure pattern of patients with resistant hypertension. Hypertension 2005; 46: 1053-1059.

27 Uzu T, Kimura G. Diuretics shift circadian rhythm of blood pressure from nondipper to dipper in essential hypertension. Circulation 1999; 100: 1635-1638.

28 Hermida RC, Calvo C, Ayala DE, Domínguez MJ, Covelo M, Fernández JR, Mojón A, López JE. Administration time-dependent effects of valsartan on ambulatory blood pressure in hypertensive subjects. Hypertension 2003; 42: 283-290.

29 Kuroda T, Kario K, Hoshide S, Hashimoto T, Nomura Y, Saito Y, Mito H, Shimada K. Effects of bedtime vs morning administration of the long-acting lipophilic angiotensinconverting enzyme inhibitor trandolapril on morning blood pressure in hypertensive patients. Hypertens Res 2004; 27: 15-20.

30 Linsell CR, Lightman SL, Mullen PE, Brown MJ, Causon RC. Circadian rhythms of epinephrine and norepinephrine in man. J Clin Endocrinol Metab 1985; 60: 1210-1215.

31 Gould BA, Mann S, Kieso H, Subramanian VB, Raftery EB. The 24-h ambulatory blood pressure profile with verapamil. Circulation $1982 ; 65: 22-27$.

32 Caruana M, Heber M, Brigden G, Raftery EB. Assessment of 'once daily' verapamil for the treatment of hypertension using ambulatory, intra-arterial blood pressure recording. Eur J Clin Pharmacol 1987; 32: 549-553.

33 Lonn E, Shaikholeslami R, Yi Q, Bosch J, Sullivan B, Tanser P, Magi A, Yusuf S. Effects of ramipril on left ventricular mass and function in cardiovascular patients with controlled blood pressure and with preserved left ventricular ejection fraction: a substudy of the Heart Outcomes Prevention Evaluation (HOPE) Trial. J Am Coll Cardiol 2004; 43: 2200-2206.

34 Black HR, Elliott WJ, Grandits G, Grambsch P, Lucente T, Neaton JD, Grimm Jr RH, Hansson L, Lacourcière $Y$, Muller JE, Sleight $P$, Weber MA, White WB, Williams GH, Wittes J, Zanchetti A, Anders RJ, CONVINCE Research Group. Results of the controlled onset Verapamil investigation of cardiovascular endpoints (CONVINCE) trial by geographical region. J Hypertens 2005; 23: 1099-1106.

35 Black HR, Elliott WJ, Grandits G, Grambsch P, Lucente T, White WB, Neaton JD, Grimm Jr RH, Hansson L, Lacourciere Y, Muller J, Sleight P, Weber MA, Williams G, Wittes J, Zanchetti A, Anders RJ, CONVINCE Research Group. Principal results of the controlled onset verapamil investigation of cardiovascular end points (CONVINCE) trial. JAMA 2003; 289: 2073-2082.

36 Portaluppi F, Smolensky MH. Perspectives on the chronotherapy of hypertension based on the results of the MAPEC study. Chronobiol Int 2010; 27: 1652-1667. 